\title{
Experiencias
}

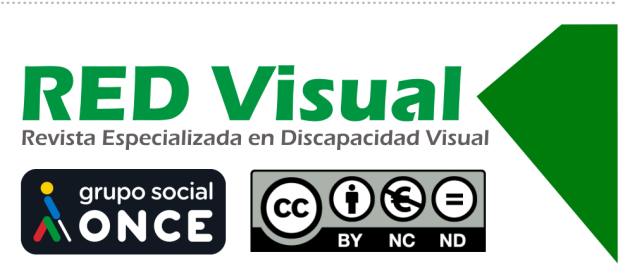

Recepción: 31-05-2021 Aceptación: 29-07-2021

\section{El ciclo de las rocas en las manos del alumnado con discapacidad visual. Taller de geología inclusiva "Ahora toca... la roca»}

The rock cycle in the hands of visually impaired pupils. Inclusive geology workshop "Ahora toca... la roca»

\author{
F. Muñiz Guinea, M. Martín Oria, M. V. Durán Labrador, \\ M. J. Narváez Vargas, Z. Belaústegui Barahona, I. González-Neira, \\ C. Barba Brioso, A. Romero Baena, J. Martinell Callicó
}

\section{Resumen}

En este trabajo se describe el taller de geología inclusiva «Ahora toca... la roca», realizado en el marco colaborativo entre la Universidad de Sevilla (US), la Universidad de Barcelona (UB) y la Organización Nacional de Ciegos Españoles (ONCE). La actividad se realizó en el Centro de Recursos Educativos de la ONCE de Sevilla (CRE) para estudiantes con discapacidad visual (DV) de secundaria y bachillerato pertenecientes a las comunidades autónomas de Andalucía y Extremadura. La experiencia se desarrolló dentro del Servicio de Educación Combinada Grupal (SECG) «iPonte a punto!» que ofrece la ONCE. El taller trató sobre el ciclo de las rocas; en particular, los procesos geológicos por los que atraviesan las rocas para transformarse en alguno de los tres tipos básicos: ígneas, sedimentarias o metamórficas. El alumnado participante en el taller, a través de la manipulación sistemática y dirigida pudo comprobar y así afianzar mejor los conocimientos acerca de la transformación que sufren las rocas. El alto grado de participación y la positiva valoración por parte del alumnado estuvieron motivados por el detallado diseño, la adaptación de los contenidos desde la perspectiva de la percepción háptica o tacto activo, la motivación del equipo educativo y su carácter multidisciplinar. 


\title{
Palabras clave
}

Discapacidad visual. Propiedades hápticas. Divulgación. Geología inclusiva. Ciclo de las rocas.

\begin{abstract}
This paper describes the inclusive geology workshop «Ahora toca... la roca», carried out in a collaborative framework among the University of Seville (US), the University of Barcelona (UB) and the Spanish National Organisation for the Blind (ONCE). The activity was carried out at the ONCE Educational Resources Centre in Seville (CRE) for visually impaired high school pupils from the Autonomous Regions of Andalusia and Extremadura. The experience was implemented within the Combined Group Education Service (SECG) «iPonte a punto!» offered by the ONCE. The workshop dealt with the rock cycle; in particular, the geological processes that rocks go through to become one of the three basic types: igneous, sedimentary or metamorphic. Through systematic and guided handling, the pupils participating in the workshop were able to verify and thus consolidate their knowledge of the transformation undergone by rocks. The high level of participation and the positive assessment by the pupils were the result of the detailed design, the adaptation of the contents from the perspective of haptic perception or active touch, the motivation of the educational team and its multidisciplinary character.
\end{abstract}

\section{Key words}

Visual impairment. Haptic properties. Dissemination. Inclusive geology. Rock cycle.

\section{Introducción}

En una sociedad dominada por la globalización de la información, el acceso al conocimiento científico por parte de la misma no es completo, a pesar de que es un derecho universal (García-Frank et al., 2014; García-Frank y Gómez-Heras, 2016; Muñiz et al., 2018). La interacción de los avances científicos con la sociedad no se puede considerar plena si no llega a todos sus colectivos, de manera especial a las personas con necesidades educativas especiales (NEE) y/o diferentes tipos de discapacidad, por ejemplo, la intelectual (García-Frank et al., 2014) o visual (Muñiz et al., 2018).

Uno de los principales retos de la sociedad para el siglo XXI es la alfabetización científica de su conjunto (Gil y Vilches, 2001; Pedrinaci et al., 2013; García-Frank et al., 
2019). Para ello, en el caso de grupos de personas con NEE, como las personas con discapacidad visual (DV), es esencial la adaptación tanto de las actividades como del material y metodología a usar bajo la óptica del Diseño Universal (Fesharaki, et al., 2016; Ramos et al., 2016; García-Frank y Fesharaki, 2016; Muñiz et al., 2018; Fesharaki et al., 2018; García-Frank et al., 2019).

Las personas con DV encuentran en la percepción háptica (táctil o estática y cinestésica o dinámica) y en el oído su principal forma de aprehensión de la información y de aprendizaje (Lucerga, 1993; Ballesteros, 1993, 1999; Lafuente, 2017). Entendemos por percepción háptica un sistema de percepción, integración y asimilación de sensaciones, del volumen y forma de los objetos a través de la información sensorial propioceptiva y táctil obtenida por el sujeto de forma activa e intencional (Lafuente, 2017). Partiendo de esta premisa, el taller se diseñó adaptando el material, así como su desarrollo en torno a la percepción háptica o tacto activo, principalmente, y al sentido auditivo.

Este equipo educativo ya ha realizado otras actividades para la enseñanza/divulgación de las Ciencias de la Tierra adaptadas a discentes con deficiencia visual: Taller de geología (fósiles y rocas) dentro de las Jornadas lúdicas para alumnos de tercer ciclo de primaria y primero de secundaria, organizadas por el CRE de la ONCE en Sevilla y el área de Cristalografía y Mineralogía de la Universidad de Sevilla (US) y celebradas en febrero de 2016; y Taller-Exposición «Mineralogía con tacto», desarrollado dentro de las Actividades de Divulgación Científica del VI Plan Propio de Investigación y Transferencia 2017 de la US, organizado por el Departamento de Cristalografía, Mineralogía y Química Agrícola y el CRE de Sevilla, y realizado en las instalaciones este último en septiembre de 2017.

En este sentido, y con la necesidad de dar continuidad a las actividades científicas inclusivas ya iniciadas en materia de Ciencias de la Tierra, desde el Departamento de Cristalografía, Mineralogía y Química Agrícola de la US y en colaboración con el CRE de Sevilla, el Departamento de Dinámica de la Tierra y del Océano de la Universidad de Barcelona (UB) y Paleonexia, de Lepe (Huelva), se ha realizado el proyecto «Ahora toca... la roca», con el objetivo principal de reforzar los conocimientos que han adquirido sobre las rocas, a partir del ciclo que las relaciona; es decir, que logren entender y aprender, de forma háptica y activa, sobre los procesos geológicos por los que atraviesan las rocas para transformarse en alguno de los tres tipos de roca: ígneas, sedimentarias o metamórficas. 
Figura 1. Carátula del proyecto

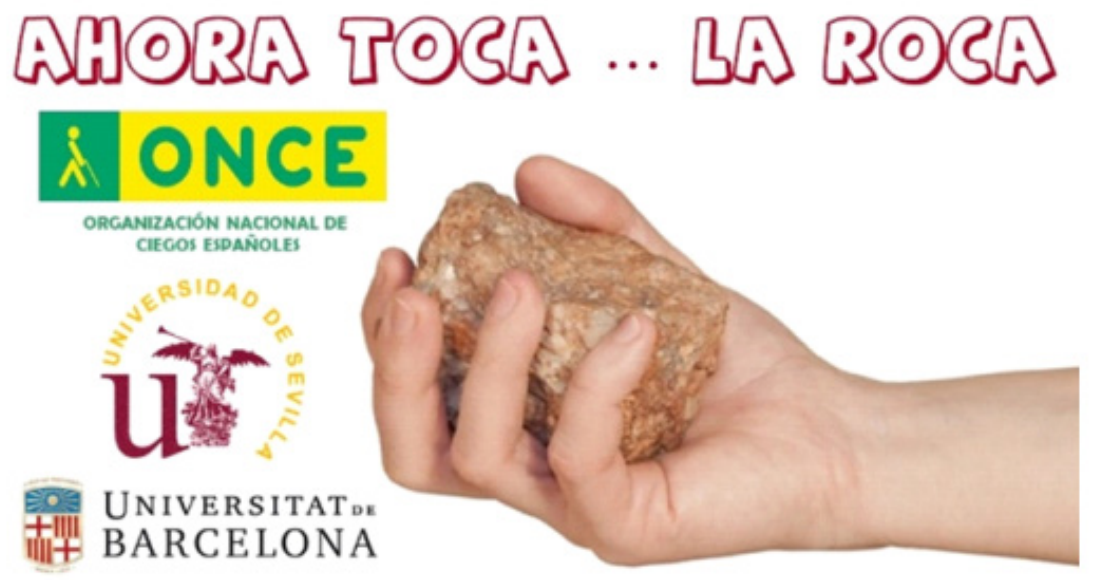

PALEONEXIA

\section{Cronología, material y metodología}

Los aspectos relativos a la planificación, diseño y ejecución del taller fueron realizados entre los meses de octubre de 2018 y septiembre de 2019 por un equipo conformado por nueve personas: dos profesores y una profesora del Área de Cristalografía y Mineralogía de la US, dos profesores del Departamento de Dinámica de la Tierra y del Océano de la UB, dos especialistas del Área de Ciencias del CRE de la ONCE en Sevilla y dos geólogas de Paleonexia (a partir de aquí nos referiremos al conjunto como «equipo educativo»).

La actividad se realizó el día 10 de septiembre de 2019, con una duración de dos horas y media, en la sede del CRE de la ONCE en Sevilla y dentro del SECG anual con título genérico «iPonte a punto!». Participaron once estudiantes (nueve con ceguera total y dos con deficiencia visual severa) de diferentes niveles académicos: uno de $6.0^{\circ}$ de EP, uno de $2 .^{\circ}$ de la ESO, tres de $3 .^{\circ}$ de la ESO, cuatro de $4 . .^{\circ}$ de la ESO, uno de $1 . .^{\circ}$ de bachillerato y uno de $2 .^{\circ}$ de bachillerato, con edades comprendidas entre los 11 y 19 años y provenientes de las comunidades autónomas de Andalucía y Extremadura.

\subsection{Cronograma de la actividad}

- En octubre de 2018 se llevaron a cabo reuniones entre el profesorado de la US y la responsable del Área de Ciencias del CRE para establecer la fecha de la actividad, 
el marco donde realizarla, el nivel de los participantes, así como las estrategias de cara al diseño de los contenidos, adaptación curricular y temporalización de la actividad.

- Entre noviembre y diciembre de 2018 se elaboró la memoria del proyecto con el título «Ahora toca... la roca». Se presentó el 28 de enero de 2019 para optar a las Ayudas para Actividades de Divulgación Científica en el marco del VI Plan Propio de Investigación y Transferencia 2019 de la US. El proyecto fue resuelto y aprobado favorablemente el 20 de abril de 2019.

- En los meses restantes hasta la ejecución de la actividad, 10 de septiembre de 2019, se realizaron las siguientes fases:

- Selección, discusión, redacción y adaptación de los textos científicos para su transcripción al sistema braille. La transcripción la realizó la responsable del Área de Ciencias del CRE de la ONCE en Sevilla.

- Elección de los materiales geológicos a usar (rocas y sedimentos, así como fósiles originales y réplicas). Estos materiales fueron aportados para la ocasión por los fondos existentes en el laboratorio de geología del Área de Cristalografía y Mineralogía en la Facultad de Ciencias de la Educación de la US, por el Departamento de Dinámica de la Tierra y del Océano de la UB y por Paleonexia. El sedimento fue recolectado por profesores de la US y UB de yacimientos del Plioceno-Cuaternario del término municipal de Lepe (Huelva), y su selección mediante tamizado se realizó en las dependencias de Paleonexia en Lepe.

- Selección y adquisición de material a usar durante el desarrollo de la actividad: cajas de cartón de diferentes tamaños, botes de cristal, bandejas y cajas de plástico de diferentes tamaños, slimes, toallitas húmedas para manos y rollos de papel para limpieza.

\subsection{El material usado}

Texto científico. Partiendo del objetivo principal de la actividad, enseñar de una manera accesible al alumnado con DV el ciclo de las rocas, se elaboró un texto para cada alumno (transcrito al braille). En este texto se contemplaron las definiciones de términos geológicos básicos (ver Tabla 1). 
Tabla 1. Términos geológicos y sus definiciones usadas

\begin{tabular}{|c|c|}
\hline Material & Descripción \\
\hline Mineral & $\begin{array}{l}\text { Sustancias naturales, sólidas, inorgánicas, homogéneas, con una } \\
\text { composición química determinada y una estructura cristalina } \\
\text { estable dentro de unos límites fisicoquímicos. }\end{array}$ \\
\hline Roca & Material sólido heterogéneo compuesto de uno o varios minerales. \\
\hline Sedimento & $\begin{array}{l}\text { Materiales no consolidados, que sufren un transporte y son acumu- } \\
\text { lados tanto en la superficie terrestre como en el fondo marino. }\end{array}$ \\
\hline Rocas sedimentarias & $\begin{array}{l}\text { Las más comunes son las detríticas (clasificadas por el tamaño de } \\
\text { grano o del sedimento). }\end{array}$ \\
\hline Rocas metamórficas & $\begin{array}{l}\text { Cualquier roca sometida a intensas presiones y temperaturas, que } \\
\text { sufre cambios en sus minerales y se transforma loen un nuevo tipo } \\
\text { de roca. }\end{array}$ \\
\hline Rocas ígneas & $\begin{array}{l}\text { Formadas a partir del enfriamiento de rocas fundidas (magmas). } \\
\text { Los magmas pueden enfriarse: } \\
\text { - De manera rápida en la superficie de la Tierra mediante la activi- } \\
\text { dad volcánica (rocas ígneas volcánicas). } \\
\text { - Lentamente en el interior, originando grandes masas de rocas } \\
\text { llamadas rocas ígneas plutónicas. } \\
\text { - Cuando se enfrían en grietas de la corteza forman las rocas } \\
\text { ígneas filonianas. }\end{array}$ \\
\hline
\end{tabular}

Sedimentos. El sedimento se clasificó según el tamaño de grano en diferentes cajas para que los participantes pudieran introducir las manos, manipularlo y analizarlo con facilidad.

- Los tamaños grava y arena gruesa se obtuvieron a partir del tamizado de material procedente de un yacimiento del periodo Cuaternario en el paraje conocido como Presa de Los Machos, al norte de la localidad de Lepe (Huelva).

- Las arenas finas se obtuvieron de un yacimiento del Plioceno inferior dentro del núcleo urbano de Lepe.

- El tamaño limo no se pudo obtener del campo, y para simular este sedimento se usó harina. 
Figura 2. Cuatro cajas con sedimentos: grava, arena gruesa, arena fina y limo

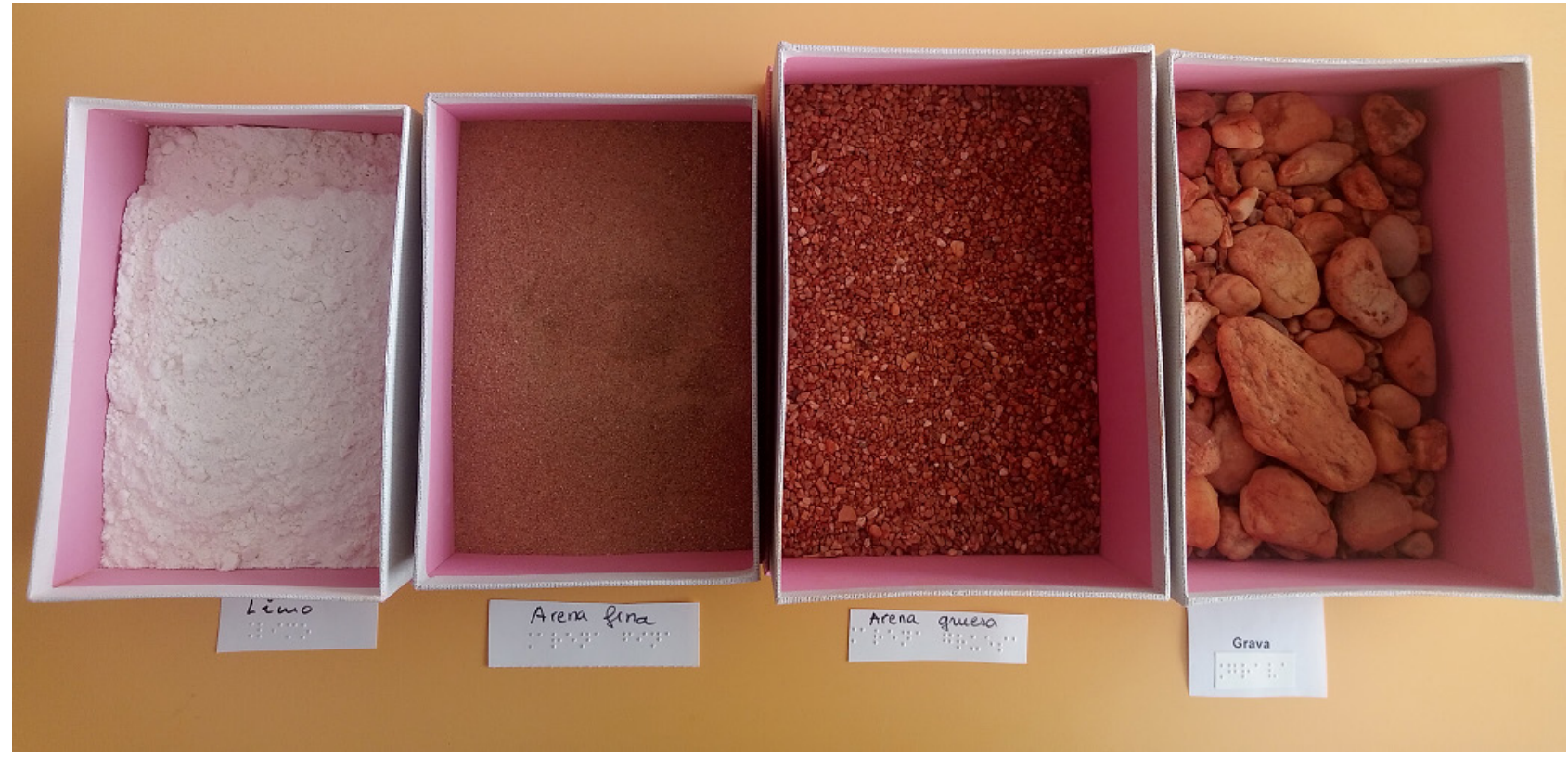

Rocas. Las rocas seleccionadas para el taller fueron las que aparecen con más frecuencia en los contenidos curriculares de la asignatura Biología y Geología de la ESO.

En cada mesa se dispuso una bandeja con ejemplares de las siguientes rocas (ver Figura 3):

- Rocas sedimentarias: conglomerado (Figura 3.I), arenisca (Figura 3.II) y caliza (Figura 3.III).

- Rocas metamórficas: pizarra (Figura 3.IV) y cuarcita (Figura. 3.V).

- Rocas ígneas: granito sin meteorizar (Figura 3.VI), meteorizado (Figura 3.VII) y costra lávica en bloque (Figura 3.VIII). Para la simulación del magma se fabricó slime (material sintético y viscoso).

Como apoyo para la explicación de las rocas ígneas volcánicas, se empleó la maqueta tiflológica de un volcán; es decir, una maqueta adaptada para que las diferentes partes del volcán puedan ser percibidas mediante el tacto por parte del alumnado. Esta maqueta fue facilitada por el Departamento de Ciencias del CRE de Sevilla. 
Figura 3. Imágenes con rocas metamórficas y sedimentarias

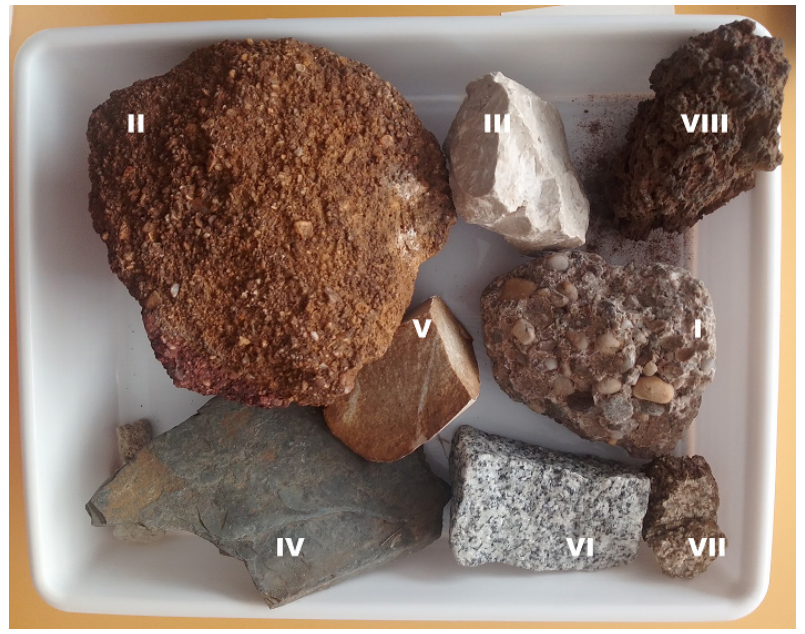

Figura 4. Imagen de maqueta de un volcán y rocas ígneas (I, basalto; II, obsidiana, y III, «bomba» volcánica)

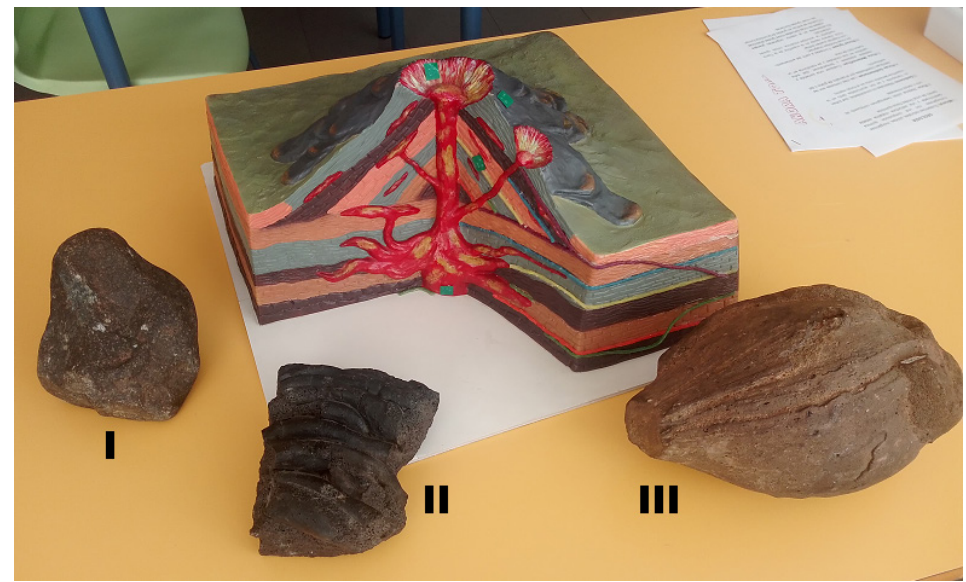

Fósiles. Los alumnos reconocieron, tocaron y discriminaron los fósiles (tanto réplicas, como originales ) y restos actuales (Figura 5):

- Réplicas de fósiles: pez Coccoderma sp. del Jurásico (Figura 5.I), huella de dinosaurio terópodo del Jurásico (Figura 5.II), trilobites Isoletus maximus del Ordovícico (Figura 5.III), trilobites Conocoriphe sp. del Cámbrico (Figura 5.IV), diente del dinosaurio Tyrannosaurus rex del Cretácico (Figura 5.V) y diente de tiburón Carcharocles megalodon del Mioceno (Figura 5.VI).

- Fósil original (molde externo) del bivalvo Acanthocardia sp. del Mioceno (Figura 5.VII). 
- Concha actual del bivalvo Acanthocardia tuberculata (Figura 5.VIII).

Figura 5. Imagen con exposición de fósiles

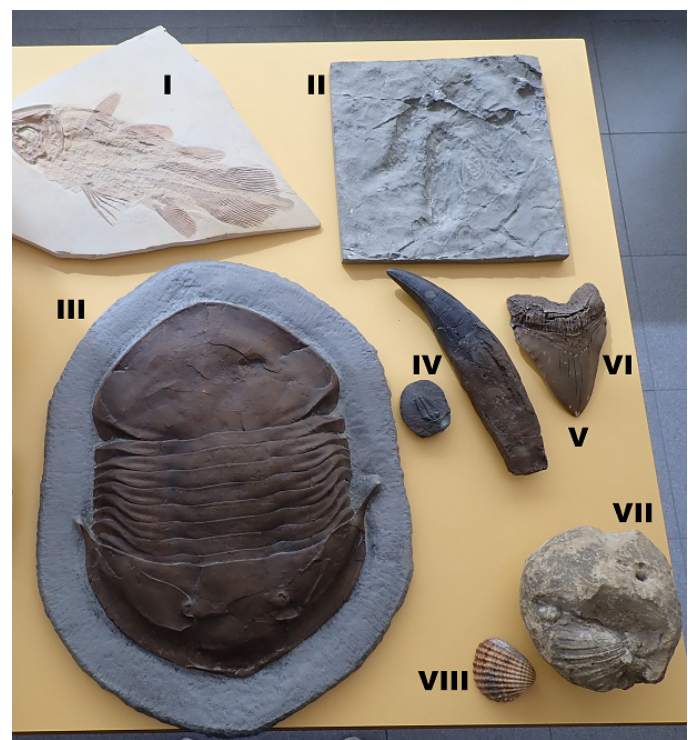

Todo este material se repartió por igual en cuatro mesas; en cada una de ellas se dispuso al alumnado (tres grupos con tres alumnos y alumnas y uno con dos alumnas), y cada grupo estuvo acompañado por dos o tres miembros del equipo educativo. Las mesas se dispusieron en forma de $U$ para conseguir una mejor fluidez en el desarrollo de la actividad y circulación del equipo educativo.

Figura 6. Imagen del aula y mesas de trabajo del taller

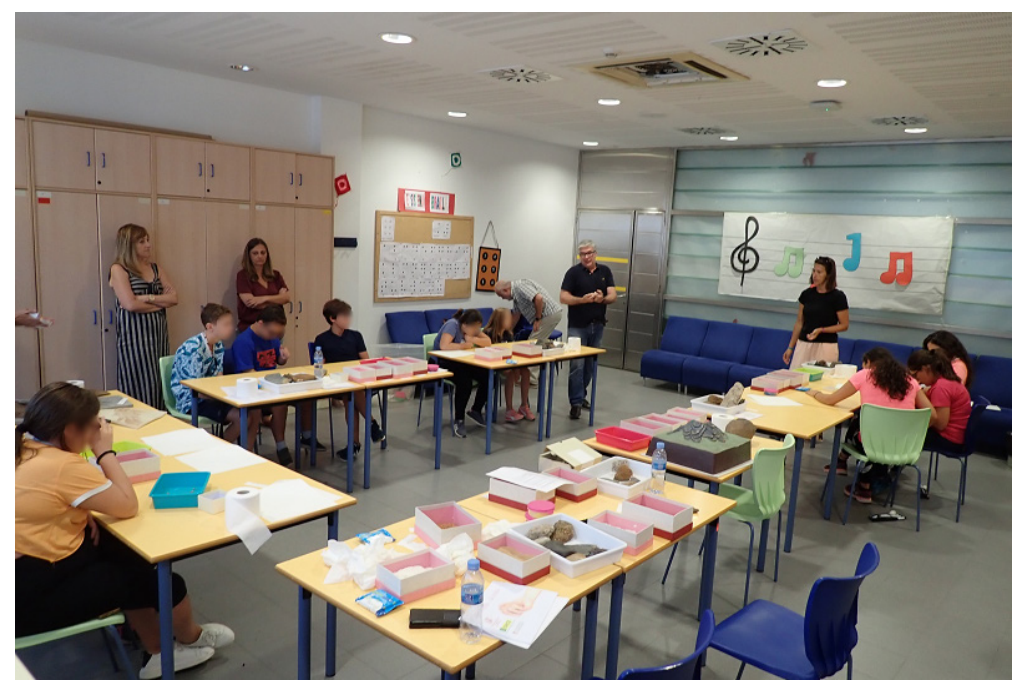

Muñiz, F., Martín, M., Durán, M.V., Narváez, M.J., Belaústegui, Z., González, I., Barba, C., Romero, A., y Martinell, J. (2021). El ciclo de las rocas en las manos del alumnado con discapacidad visual. Taller de geología inclusiva «Ahora toca... la roca». RED Visual: Revista Especializada en Discapacidad Visual, 78, 133-155. https://doi.org/10.53094/BQOF1695. 


\subsection{Metodología}

1. Se centra la actividad comentando, en líneas generales, los objetivos del taller y la temática a desarrollar.

2. Se explicitan las ideas previas del alumnado acerca de los distintos tipos de rocas y su génesis.

3. Se presenta nueva información. En este caso, esta se basó, fundamentalmente, en la génesis de los distintos tipos de rocas y en la diferenciación de las mismas.

4. Para la diferenciación de los diferentes tipos de rocas se usó: la exploración háptica, que nos permite distinguirlas en función de su forma, textura, peso, etc., así como la percepción acústica, que las diferencia en función de su posibilidad de reacción con otras sustancias, debido a su diferente composición (concretamente, se vio cómo las rocas carbonatadas reaccionan con el ácido clorhídrico y, en este proceso, se produce una efervescencia distinguible al oído).

5. Se sintetiza el proceso seguido y los aprendizajes realizados. Esta síntesis y valoración final se realiza de manera grupal y semiestructurada. Se valora el nivel de motivación del alumnado con respecto a la metodología seguida en el taller.

\section{El desarrollo del taller}

\section{Fase 1. Recordando conceptos}

El taller comenzó con una presentación de los participantes, tanto del alumnado como del equipo educativo. A continuación, se comentó el objetivo de la actividad (aprender sobre las rocas y su ciclo de una forma diferente y práctica) y un recordatorio de algunos conceptos básicos de geología desarrollados en el texto en braille facilitado a cada alumno: ¿qué es la geología?, ¿qué es un mineral?, ¿qué es una roca?, y tipos básicos de rocas (sedimentarias, ígneas y metamóficas). Para generar un ambiente participativo y distendido, favoreciendo el estímulo inclusivo, cada definición fue leída en voz alta por un alumno (Figura 7) y se procedió a resolver las dudas. 
Figura 7. Participantes del taller leyendo contenidos teóricos a trabajar

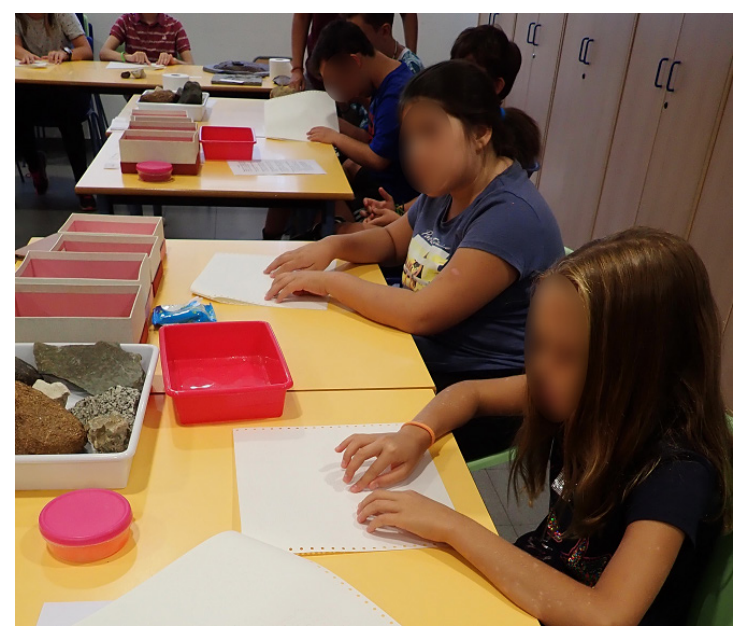

\section{Fase 2. El sedimento}

Una alumna leyó la definición de sedimento y se resolvieron dudas. Se explicó que los sedimentos se clasifican según el tamaño de los granos (grava, arena gruesa, arena fina, limo).

El equipo educativo acercó las cajas con los diferentes tipos de sedimentos, así como las etiquetas (en braille) con sus nombres. Los participantes del taller, correlacionaron cada una de las cajas con sus etiquetas correspondientes.

Figura 8. Alumnado analizando y comparando sedimentos de distintos tamaños

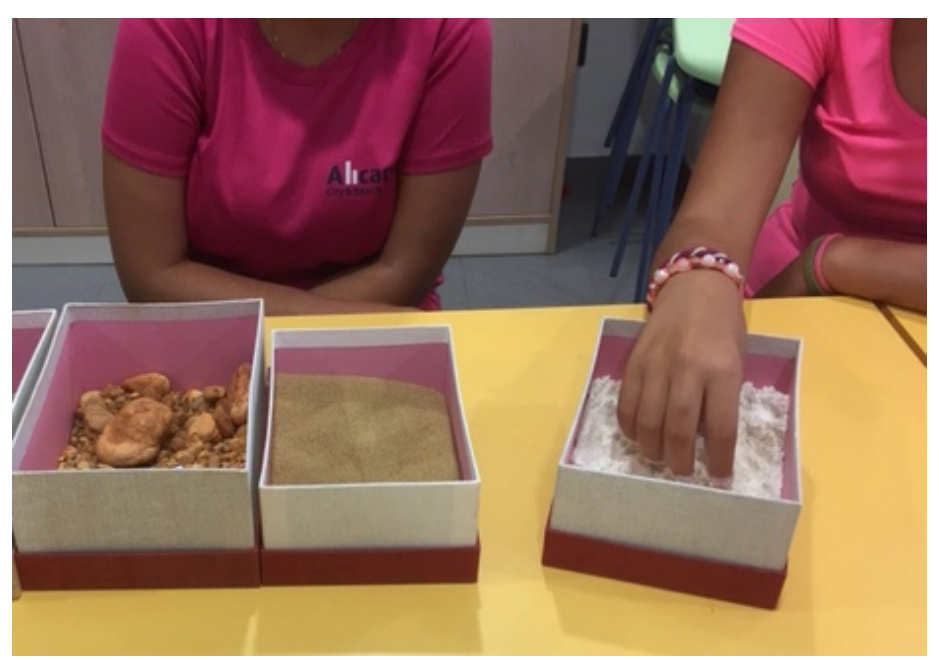

Muñiz, F., Martín, M., Durán, M.V., Narváez, M.J., Belaústegui, Z., González, I., Barba, C., Romero, A., y Martinell, J. (2021). El ciclo de las rocas en las manos del alumnado con discapacidad visual. Taller de geología inclusiva «Ahora toca... la roca». RED Visual: Revista Especializada en Discapacidad Visual, 78, 133-155. https://doi.org/10.53094/BQOF1695. 


\section{Fase 3. De los sedimentos a rocas sedimentarias}

Se leyó la definición de roca sedimentaria; se resolvieron las dudas. A continuación, el equipo educativo colocó la bandeja libre en cada mesa y les fueron colocando una a una las rocas: primero el conglomerado, luego la arenisca y, por último, la caliza. En este punto, relacionaron y compararon manipulativamente el tamaño de los granos/ clastos entre cada roca y, luego, con el sedimento manipulado anteriormente.

\section{Fase 4. Rocas con fósiles}

El equipo educativo explicó a todos los grupos qué es un fósil: restos de organismos (animales y plantas), o de su actividad biológica (por ejemplo, huellas, madrigueras, coprolitos, etc.), que poblaron la tierra en épocas pasadas y que se han conservado en las rocas. Se resolvieron dudas y, seguidamente, se fue repartiendo por las mesas el material de «fósiles» hasta que todos los hubieron manipulado.

Figura 9. Reconociendo manipulativamente un fósil de bivalvo Acanthocardia

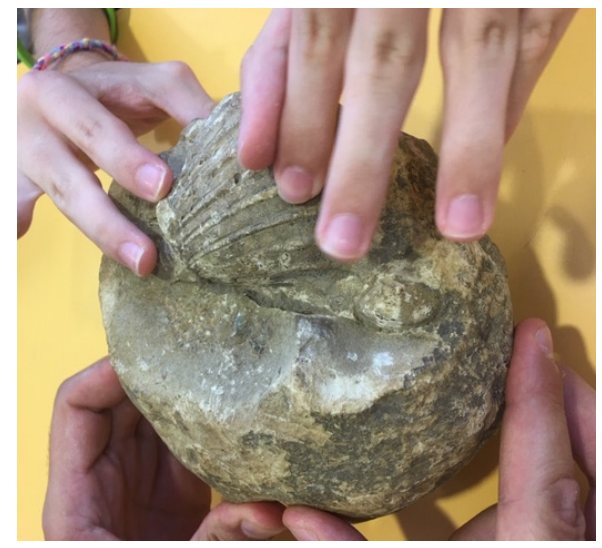

\section{Fase 5. Escuchando una roca}

Se explicó que hay algunas rocas compuestas, íntegra o parcialmente, por carbonato cálcico $\left(\mathrm{CaCO}_{3}\right)$, como la caliza, conglomerados con matriz carbonatada (roca sedimentaria detrítica) o el mármol (roca metamórfica).

Partiendo de su composición (carbonato cálcico), podemos reconocerlas haciéndolas reaccionar con ácido clorhídrico $(\mathrm{HCl})$. Esta reacción provoca una efervescencia que es perceptible al oído. 
Los participantes pudieron comprobarlo introduciendo en una disolución diluida de $\mathrm{HCl}$ dos tipos de rocas: pizarra y caliza (sin y con carbonato cálcico respectivamente). Así pudieron comprobar que, al introducir la segunda roca, se escuchaba una efervescencia producto de la reacción (Figuras 10 y 11).

\section{Fase 6. Rocas metamórficas}

En esta nueva fase, un alumno leyó la definición de roca metamórfica (Tabla 1); después, un integrante del equipo educativo comentó para el grupo los principales factores que intervienen (la presión y la temperatura) y se resolvieron las dudas. Para la comprensión táctil de este concepto, se tomaron como referencia dos rocas sedimentarias, una de grano muy fino (limo) y otra de grano medio (arenisca), y se les indicó que, si son sometidas a estos factores, cada una sufre una transformación a otra roca. En concreto, el limo pasa a ser una pizarra y la arenisca a cuarcita.

Figura 10. Proceso de efervescencia de la roca caliza

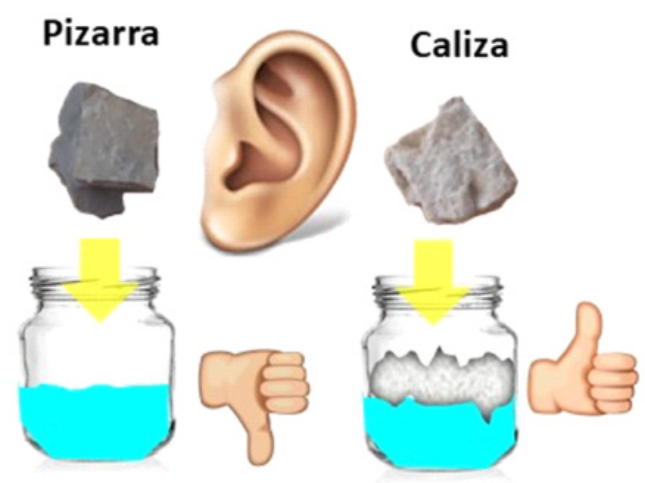

Figura 11. Escuchando las rocas calizas

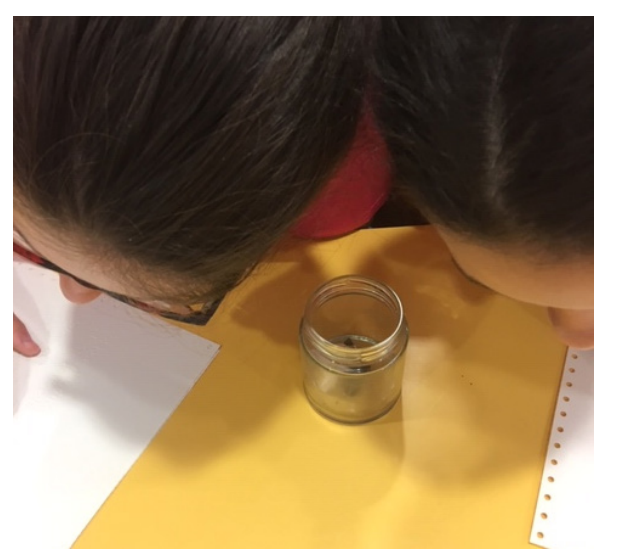

Muñiz, F., Martín, M., Durán, M.V., Narváez, M.J., Belaústegui, Z., González, I., Barba, C., Romero, A., y Martinell, J. (2021). El ciclo de las rocas en las manos del alumnado con discapacidad visual. Taller de geología inclusiva «Ahora toca... la roca». RED Visual: Revista Especializada en Discapacidad Visual, 78, 133-155. https://doi.org/10.53094/BQOF1695. 
El alumnado manipuló estas rocas metamórficas y se les indicaron los criterios para diferenciar cada una:

- La textura de la pizarra se llama foliación (concepto que proviene del término folium, que significa «hoja» en latín); es decir, que la pizarra es como un «libro» (Figura 12) conformado por pequeñas capas o láminas (hojas) que pueden llegar a separarse fácilmente.

- Para reconocer la textura de la cuarcita, primero se les dio a manipular la arenisca y comprobaron su textura granular; luego, tocaron la cuarcita y llegaron a la conclusión de que tiene una textura diferente, no foliada, y donde los granos no se perciben táctilmente.

Figura 12. Alumno tocando un fragmento de pizarra y comparándolo con las hojas de un libro

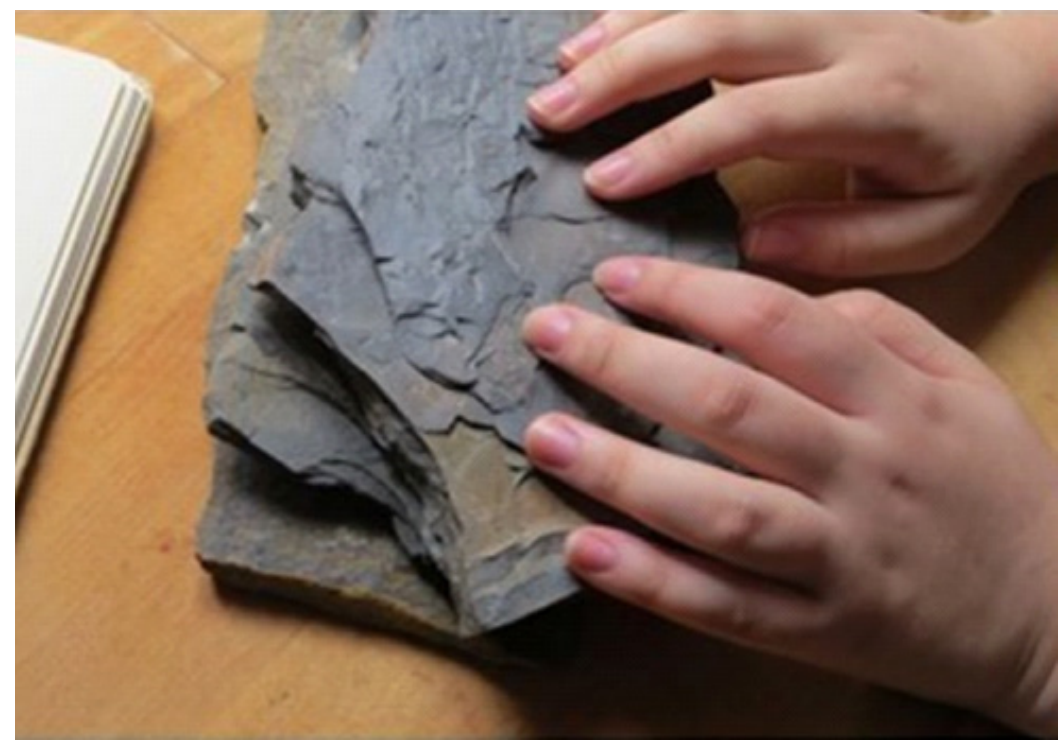

\section{Fase 7. Rocas ígneas}

Nuevamente, se comenzó leyendo y comentando la definición de roca ígnea y sus tipos en función del emplazamiento y la «velocidad» de enfriamiento (rocas plutónicas, volcánicas y filonianas). Se comentó que estas rocas pueden también someterse a presión y temperatura y sufrir transformaciones; es decir, metamorfismo. Las rocas que se trabajaron en esta fase fueron: A) de las plutónicas, el granito, y B) de las volcánicas, el basalto, la costra y la bomba lávica, la obsidiana y la pumita. 
El equipo educativo aproximó las rocas ígneas y, en primer lugar, los participantes manipularon el granito. A continuación, se les expusieron las rocas volcánicas, comprobando las diferentes texturas y el «peso» del basalto (Figura 13), la costra lávica, la obsidiana y la pumita (Figura 14).

Figura 13. Manos de alumna tocando un fragmento de basalto

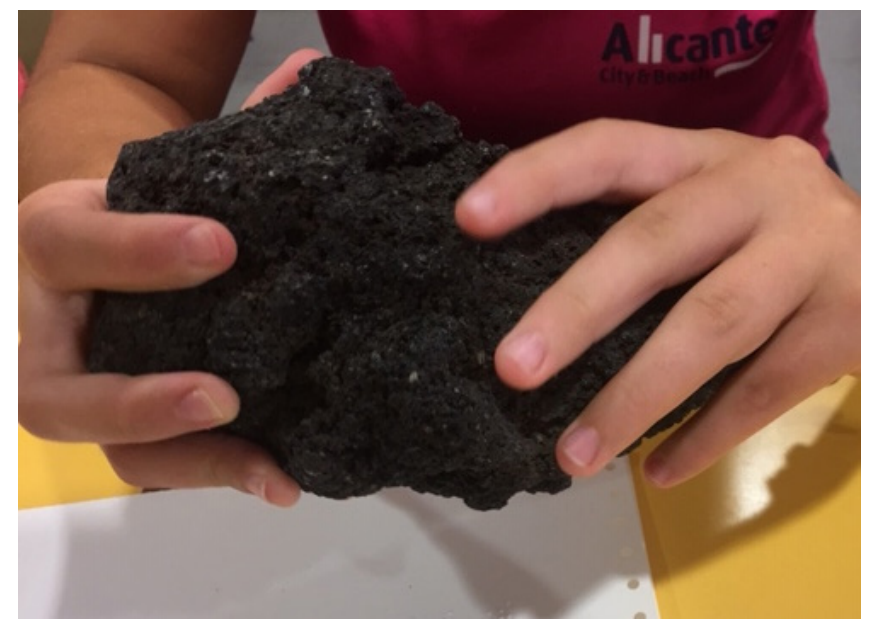

Figura 14. Manos del alumnado tocando una bomba volcánica

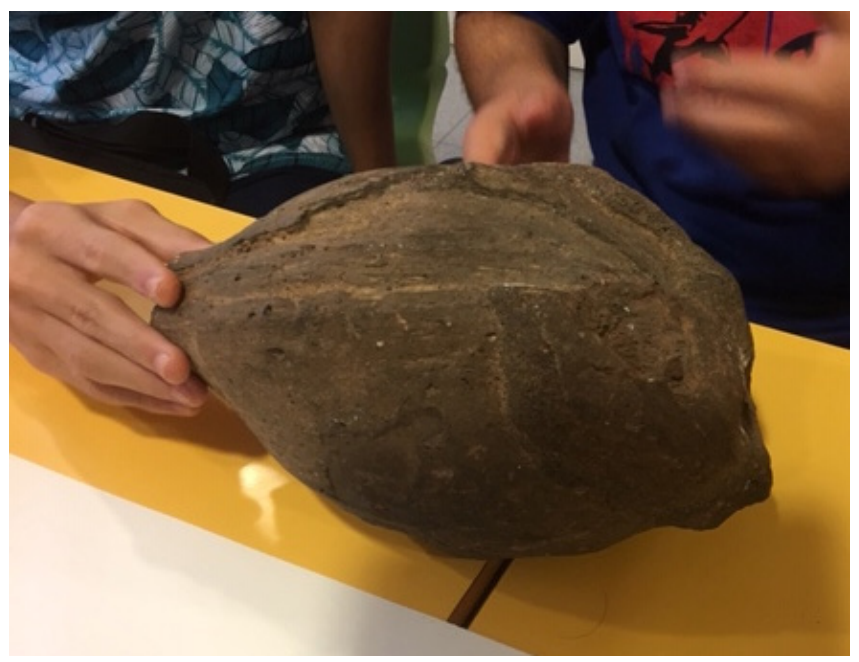

Posteriormente, se les acercó la maqueta tiflológica del volcán para reconocer sus principales partes: cámara magmática, chimenea, cráter, capas de material volcánico y coladas de lava. Paralelamente a la manipulación de la maqueta, se enseñaron varias rocas de productos volcánicos generados por la erupción: una bomba volcánica, costra lávica cordada y pumita. Algunas de las partes de la maqueta disponían de etiquetas 
LEO (elaboradas por el Área de Ciencias del CRE) con información asociada y legible con el lápiz lector óptico LEO (más información en https://cti.once.es/catalogo-comercial/ buscador-del-catalogo-comercial/view_item?prodcodid=026\&prodcodanid=2013). El lápiz, en la punta, tiene un sensor que, en contacto con la etiqueta, hace que salte un archivo de audio con la información previamente grabada en el mismo.

Otro concepto que se trabajó fue el de la viscosidad del magma. Para que el alumnado comprenda mejor este término, se empleó slime, que es un material artificial viscoso; este concepto fue entendido perfectamente, ya que se fue pasando el material de mano en mano por todos los grupos.

\section{Fase 8. De las rocas al sedimento}

Una vez reconocieron táctilmente los tipos básicos de rocas, un miembro del equipo educativo comentó que todas estas rocas pueden sufrir un proceso de alteración denominado meteorización; es decir, su descomposición en granos por la acción de diferentes agentes (agua, temperatura, organismos, etc.), y que estos granos pueden sufrir a posteriori erosión, transporte por diferentes agentes (aire, agua, la gravedad) $y$, finalmente, sedimentación, dando lugar a los sedimentos como los manipulados al principio de la actividad. Se les puntualizó que, si estos granos no sufren los procesos de erosión y transporte, darán lugar a lo que conocemos como suelo.

Para la comprensión del efecto producido por la meteorización en una roca, el equipo educativo volvió a acercar, para que tocasen, el granito usado en la fase anterior, y así recordar su compacidad (Figura 15); luego, se les cambió por un granito en estado de descomposición y fácilmente disgregable en partículas menores, es decir, sedimento (Figura 16).

Figura 15. Comprobando compacidad del granito

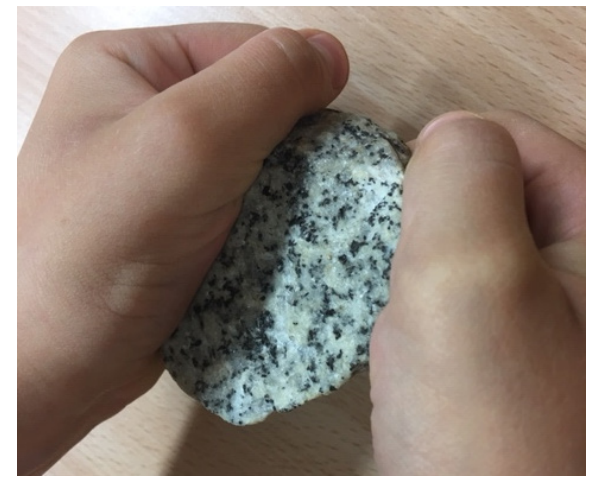

Muñiz, F., Martín, M., Durán, M.V., Narváez, M.J., Belaústegui, Z., González, I., Barba, C., Romero, A., y Martinell, J. (2021). El ciclo de las rocas en las manos del alumnado con discapacidad visual. Taller de geología inclusiva «Ahora toca... la roca». RED Visual: Revista Especializada en Discapacidad Visual, 78, 133-155. https://doi.org/10.53094/BQOF1695. 
Figura 16. Manipulando el sedimento

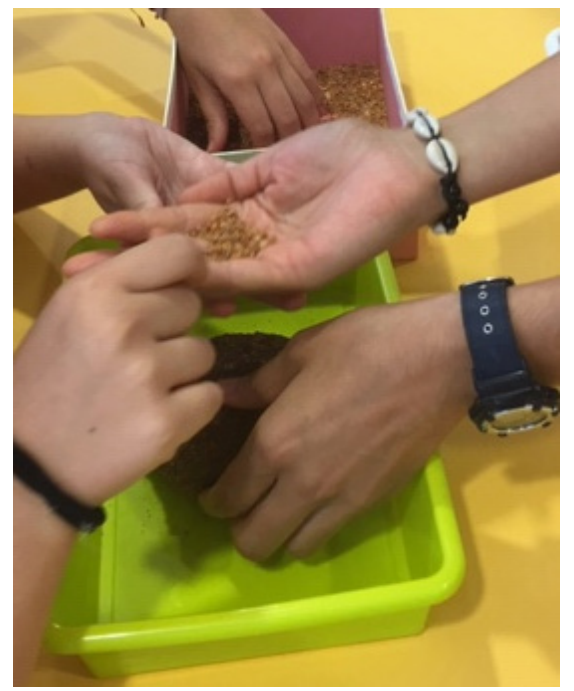

\section{Resultados}

Finalizada la actividad, se realizó una puesta en común grupal con el fin de conocer las impresiones sobre aquella por parte del alumnado. Todos los participantes manifestaron que, a pesar de haber estudiado los tipos de rocas y su ciclo en el instituto, nunca habían realizado una práctica manipulando las rocas, de ahí que resultase una experiencia muy satisfactoria para su aprendizaje por la adaptación del material, el tamaño de los grupos, la dinámica de la actividad y la implicación del equipo educativo. Por otra parte, desde el CRE, suelen realizar un cuestionario de valoración de cada actividad al finalizar «iPonte a punto!». Todos los que contestan (8 de 11) a la valoración del taller «Ahora toca...la roca» le conceden la máxima puntuación.

A continuación, pasamos a comentar los resultados observados en cada una de las fases del taller:

- Fase 1. Recordando conceptos: como se ha comentado al principio de este apartado, el alumnado reconoce haber estudiado las rocas y su ciclo. Demuestran conocer, aunque de forma somera, las cuestiones planteadas antes de la lectura de conceptos. En cuanto a los tres tipos de rocas, evidencian que, más o menos, las conocen de forma teórica, aunque no tanto los procesos que las originan, en especial, para las metamórficas y sedimentarias. Reconocen no haber trabajado nunca con muestras de ellas. 
- Fase 2. El sedimento: en esta fase, no encontraron dificultad durante la percepción táctil de los diferentes sedimentos repartidos en cajas. Asimilaron perfectamente la clasificación del sedimento por el tamaño de los granos. Una alumna, incluso, supo relacionar el sedimento que estaba manipulando con el de playas en las que había estado, en particular, las gravas con una playa llamada de «guijarros» de Estepona, en Málaga, y la arena fina con playas de Cádiz. A todos les llamó la atención el tacto del limo y su i«olor a harina»!, tanto que alguna alumna no se dejó «engañar». Se les explicó que el sedimento tamaño limo real es igual de fino y con igual textura que la harina. No hubo confusión ni dudas al enlazar los nombres de los sedimentos escritos en braille (en etiquetas) con el contenido de cada caja.

- Fase 3. De los sedimentos a las rocas sedimentarias: todo el alumnado relacionó adecuadamente cada una de las rocas sedimentarias que tocaron (diferenciando el tamaño de los clastos) con los sedimentos seleccionados por tamaños de la fase anterior: grava con conglomerado y arena gruesa con arenisca. Los conceptos técnicos de diagénesis, litificación y cementación, una vez comentados, no ocasionaron dudas a la hora de entender el proceso de formación de muchas de estas rocas. De manera particular, en una mesa sí preguntaron sobre el origen de la roca caliza (la cual se explicó más adelante).

- Fase 4. Rocas con fósiles: esta fase fue de gran disfrute por parte de los participantes pues, al igual que las rocas, la mayoría «ino había tocado nunca un fósil!». La selección de las piezas fue intencionada, pues se mostraban con un alto potencial de cara a explotar la percepción háptica. Cuatro de los fósiles (los dos trilobites, el pez y la huella de dinosaurio) simulaban estar incluidos en una superficie rocosa horizontal con diferentes relieves: positivo en el caso de los trilobites y negativo en el del pez y la huella. Al tacto activo reconocieron fácilmente la forma y sus partes anatómicas. Los dientes de tiranosaurio y megalodón reproducían las tres dimensiones. Por último, la formación del molde de un fósil, caso del molde externo de bivalvo usado, la asimilaron bien gracias a que, previamente, tocaron la concha de un bivalvo actual con similar ornamentación.

- Fase 5. Escuchando una roca sedimentaria: esta parte experimental fue muy agradecida por el alumnado, pues comentaron que el acceso a prácticas de laboratorios con productos químicos lo tienen muy limitado, y el hecho de haber manipulado (aun con ayuda) el ácido clorhídrico (HCL) y las rocas fue satisfactorio. La experiencia de usar el sentido del oído para reconocer el tipo de rocas sedimen-

Muñiz, F., Martín, M., Durán, M.V., Narváez, M.J., Belaústegui, Z., González, I., Barba, C., Romero, A., y Martinell, J. (2021). El ciclo de las rocas en las manos del alumnado con discapacidad visual. Taller de geología inclusiva «Ahora toca... la roca». RED Visual: Revista Especializada en Discapacidad Visual, 78, 133-155. https://doi.org/10.53094/BQOF1695. 
tarias carbonatadas les sorprendió. Todos relacionaron la reacción efervescente de la caliza en el ácido clorhídrico con la de comprimidos que tienen el mismo efecto en presencia de agua.

- Fase 6. Rocas metamórficas: el alumnado comprendió bien que las rocas se transforman cuando son sometidas a grandes cambios de presión y temperatura. Para asimilar conceptos, algunos monitores incitaron a alumnos a que intentaran «aplastar» con sus manos una de las rocas: comprobaron, así, la dificultad y, sobre todo, infirieron la presión a la que tienen que someterse las rocas para ser transformadas. Este proceso de metamorfismo (por presión) lo entendieron una vez que manipularon la pizarra, a partir de su textura foliada y la comparación con las hojas de un libro. No encontraron dificultad en entender la transformación de una arenisca en una cuarcita, pero sí la distinción de esta última con otras rocas de textura similar.

- Fase 7. Rocas ígneas: el alumnado no tuvo complicaciones en ir reconociendo y asimilando algunas características significativas entre las rocas volcánicas empleadas en la actividad, a las que se refirieron como: el «peso (densidad) ligero» y el «tacto (textura) áspero» de la costra lávica; el «peso alto» del basalto; el «poco peso» de la pumita, y la «sensación de vidrio (textura vítrea)» y «fría» de la obsidiana. Para algunas de estas rocas, preguntaron sobre el origen y el porqué de estas características. Por otro lado, la maqueta tiflológica del volcán facilitó mucho la comprensión de la estructura de un volcán tipo (estratovolcán) y alguno de sus productos volcánicos, como la lava y la bomba volcánica, cuya morfología ovoidal la relacionaron con un «balón de rugby» y un «melón». En esta fase, la mayor dificultad estuvo en el reconocimiento háptico de las características texturales que definen al granito, usado como ejemplo de roca plutónica. No pudieron reconocer el tamaño de los cristales que lo componen (cuarzo, feldespato y mica) y que definen su «textura (holo)cristalina fanerítica», es decir, un enfriamiento lento de las rocas plutónicas hace que, normalmente, el porcentaje de cristales en ellas sea más del $90 \%$.

- Fase 8. De la roca al sedimento: en esta última fase, el granito fue de gran importancia para que el alumnado comprendiera el proceso de meteorización, cuyo concepto teórico entendió bien y, en consecuencia, la génesis del sedimento. Una vez comprobado que era imposible fragmentar con las manos el granito que tocaron con anterioridad, les resultó «impactante» poder desgranar con muchísi-

Muñiz, F., Martín, M., Durán, M.V., Narváez, M.J., Belaústegui, Z., González, I., Barba, C., Romero, A., y Martinell, J. (2021). El ciclo de las rocas en las manos del alumnado con discapacidad visual. Taller de geología inclusiva «Ahora toca... la roca». RED Visual: Revista Especializada en Discapacidad Visual, 78, 133-155. https://doi.org/10.53094/BQOF1695. 
ma facilidad la misma roca (otro ejemplar) una vez que esta estuvo sometida a los agentes de alteración. La comprensión de que este «experimento» les conducía al principio del taller, al sedimento, demostraba, sin duda, que así se cerraba «el ciclo de las rocas» (Figura 17).

Figura 17. Esquema del ciclo de las rocas

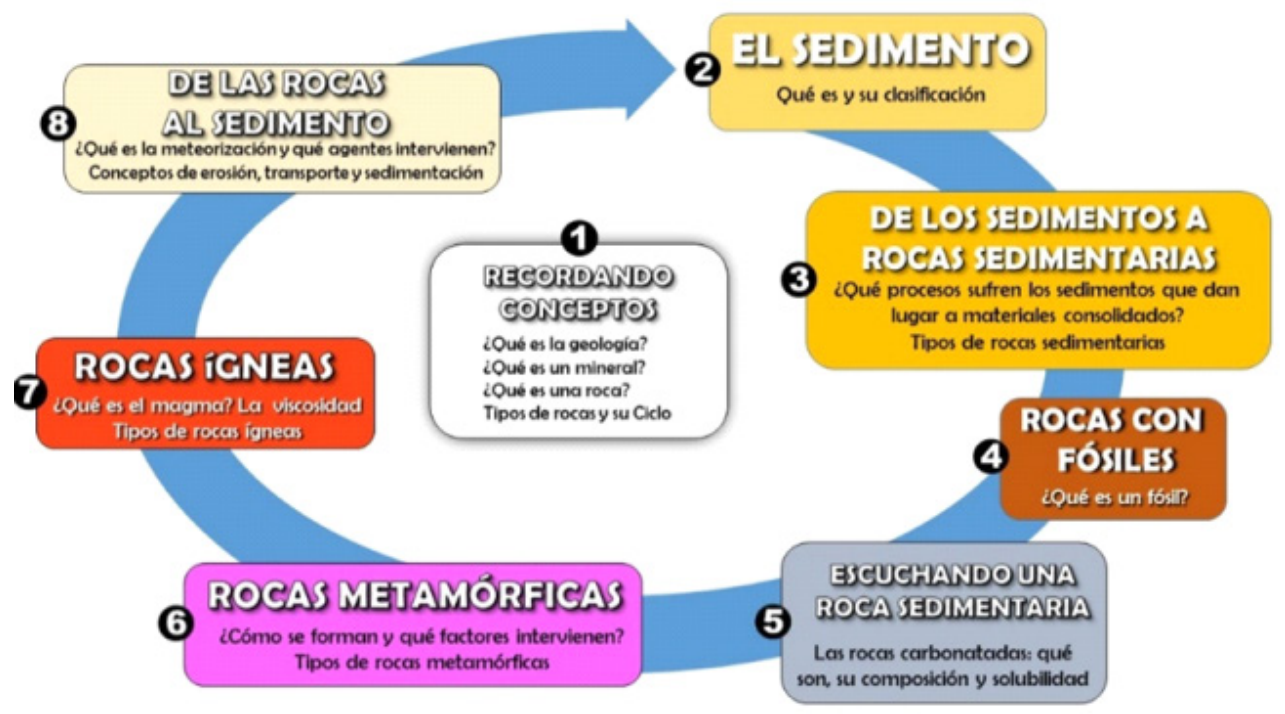

\section{Conclusiones}

Las rocas y su ciclo son parte de los contenidos geológicos que el alumnado estudia en materias curriculares impartidas durante diferentes niveles de la ESO y algunos itinerarios de bachillerato. Por lo general, en el ámbito del aula-laboratorio, y para estos niveles académicos, no está, o lo está mínimamente, normalizada la alfabetización en estos temas de las ciencias de la Tierra para alumnado con DV, sobre todo en lo que a prácticas de laboratorio se refiere. Esta circunstancia se debe, entre otras carencias, a la falta de una adaptación desde el diseño universal. En este sentido, la experiencia descrita en este trabajo es novedosa, pues propone una detallada adaptación del diseño de una actividad y del material, focalizada hacia la percepción háptica, o tacto activo, de las propiedades y características de las rocas y su ciclo. El resultado de la experiencia viene a demostrar que es posible que estos alumnos con DV (y extrapolable al alumnado sin ella) fijen y comprendan conocimientos relativos a las ciencias de la Tierra, pero, siempre teniendo en cuenta la adaptación de los contenidos y la motivación, tanto de los estudiantes como del profesorado. 


\section{Agradecimientos}

Proyecto financiado por el VI Plan Propio de Investigación y Transferencia de la Universidad de Sevilla. Los autores agradecen las facilidades ofrecidas por la dirección del Centro de Recursos Educativos de la ONCE en Sevilla. Agradecer también al Área de Cristalografía y Mineralogía de la US todo el material geológico facilitado. Muchas gracias a la Dra. Meritxell Aulinas (Departamento de Mineralogía, Petrología y Geología Aplicada de la UB) y a Fadoua Zohra (Litoteca UB) por todo el material prestado y relacionado principalmente con las rocas ígneas. Al Grupo de Investigación RNM 293 «Geomorfología Ambiental y Recursos Hídricos» de la Universidad de Huelva.

\section{Referencias bibliográficas}

Ballesteros, S. (1993). Percepción háptica de objetos y patrones realzados: una revisión [PDF]. Psicothema, 5(2), 311-321.

Ballesteros, S. (1999). Evaluación de las habilidades hápticas [Word]. Integración: revista sobre ceguera y deficiencia visual, 31, 5-15.

Fesharaki, O., García-Frank, A., Iglesias, N., Gómez-Heras, M., Martín-Perea, D.M., y Rico, D. (2016). Diseño universal y materiales multisensoriales en las actividades de divulgación de Geodivulgar con la asociación Ciencia sin Barreras [PDF]. Geo-Temas, 16(1), 729-732.

Fesharaki, O., Iglesias, N., Durán, A., y García-Frank, A. (2018). Talleres de mineralogía adaptados para público diverso [PDF]. Macla, 23, 2 pp.

García-Frank, A., y Fesharaki, O. (2016). Rompiendo barreras en pos de una ciencia accesible [PDF]. En M. González, A. Baratas y A. Brandi (eds.), Jornadas sobre Investigación y Didáctica en ESO y Bachillerato; Actas del IV Congreso de Docentes de Ciencias de la Naturaleza (pp. 15-25). Santillana.

García-Frank, A., Fesharaki, O., y Rodrigo, A. (2019). Innovación en la divulgación de la Geología: propuestas inclusivas hechas por estudiantes para estudiantes [PDF]. Enseñanza de las Ciencias de la Tierra, 27(1), 116-118. 
García-Frank, A., y Gómez-Heras, M. (2016). Hacia una ciencia accesible [recurso web]. Fundación para el Conocimiento madri+d.

García-Frank, A., Pérez, R., Espín, B., Benito, P., De Pablo, L., Gómez-Heras, M., Sarmiento, G. N., Canales, M. L., González, L., Muñoz, M. B., García, R., Hontecillas, D., Ureta, M.S., y Del Moral, B. (2014). Divulgación de la Geología: nuevas estrategias educativas para alumnos con necesidades educativas especiales por discapacidad intelectual [PDF]. El CSIC en la escuela: investigación sobre la enseñanza de la ciencia en el aula, 10, 56-67.

Gil, D., y Vilches, A. (2001). Una alfabetización científica para el siglo XXI: obstáculos y propuestas de actuación [PDF]. Investigación en la Escuela, 43, 27-37.

Lafuente, M.Á. (2017). Educación Inclusiva: personas con discapacidad visual [recurso web]. Ministerio de Educación, Instituto de Tecnologías Educativas.

Lucerga, R. (1993). Palmo a palmo: la motricidad fina y la conducta adaptativa a los objetos en los niños ciegos [Word]. Organización Nacional de Ciegos Españoles.

Muñiz, F., Romero, A., Martínez, R., Durán, M.V., Narváez, M. J., Lozano, O., Miras, A., y Martín, M. (2018). Mineralogía con tacto [PDF]. Enseñanza de las Ciencias de la Tierra, 26(3), 366-374.

Pedrinaci, E., Alcalde, S., Alfaro, P., Almodóvar, G. R., Barrera, J. L., Belmonte, Á., Brusi, D., Calonge, A., Cardona, V., Crespo-Blanc, A., Feixas, J. C., Fernández-Martínez, E. M., González-Díez, A., Jiménez-Millán, J., López-Ruiz, J., Mata-Perelló, J.. M., Pascual, J. A., Quintanilla, L., Rábano, I., ... Roquero, E. (2013). Alfabetización en ciencias de la Tierra [PDF]. Enseñanza de las Ciencias de la Tierra, 21(2), 117-129.

Ramos, L., Castro, E., y Castro-Rodríguez, E. (2016). Instrucción en el uso de esquemas para la resolución de problemas aditivos a estudiantes con necesidades educativas especiales [PDF]. Enseñanza de las Ciencias, 34(1), 173-192. 
Fernando Muñiz Guinea. Profesor de universidad. Departamento de Cristalografía, Mineralogía y Química Agrícola, Facultad de Química, Universidad de Sevilla (US). Avda. de la Reina Mercedes, 6; 41012 Sevilla (España).Correo electrónico: fmuniz@us.es.

Mónica Martín Oria. Paleonexia. Guzmán y Zúñiga, 61-1. a D; 21440 Lepe, Huelva (España). Correo electrónico: Momao185@gmail.com.

M. ${ }^{a}$ Valle Durán Labrador. Especialista de Ciencias Experimentales del Centro de Recursos Educativos de la ONCE en Sevilla. Campo de los Mártires, 10; 41018. Sevilla (España). Correo electrónico:mvdl@once.es.

M. José Narváez Vargas. Profesora de educación secundaria del Equipo específico de apoyo educativo al alumnado con discapacidad visual (Convenio Junta-ONCE), referente de ciencias en el CRE de la ONCE en Sevilla. Campo de los Mártires, 10; 41018 Sevilla (España). Correo electrónico: mnv@once.es.

Zain Belaústegui Barahona. Profesor de universidad. Departamento de Dinámica de la Tierra y del Océano, Facultad de Ciencias de la Tierra, Instituto de Investigación de la Biodiversidad (IRBio), Universidad de Barcelona (UB). Martí i Franquès, s/n; 08028 Barcelona (España). Correo electrónico: zbelaustegui@ub.edu.

Isabel González Neira. Paleonexia. Isla Canela, blq. 5-1.o; 21440 Lepe, Huelva (España). Correo electrónico: isabelgneira@gmail.com.

Cinta Barba Brioso. Profesora de universidad. Departamento de Cristalografía, Mineralogía y Química Agrícola, Facultad de Química, Universidad de Sevilla (US). Avda. de la Reina Mercedes, 6; 41012 Sevilla (España). Correo electrónico: cbarba@us.es.

Antonio Romero Baena. Profesor de universidad. Departamento de Cristalografía, Mineralogía y Química Agrícola, Facultad de Química, Universidad de Sevilla (US). Avda. de la Reina Mercedes, 6; 41012 Sevilla (España). Correo electrónico: aromero@us.es.

Jordi Martinell Callicó. Catedrático emérito. Departamento de Dinámica de la Tierra y del Océano, Facultad de Ciencias de la Tierra, Instituto de Investigación de la Biodiversidad (IRBio), Universidad de Barcelona (UB). Martí i Franquès, s/n; 08028 Barcelona (España). Correo electrónico: jmartinell@ub.edu. 